\title{
Influência da cisplatina administrada no pré e no pós-operatório sobre a cicatrização de anastomoses colônicas em ratos
}

\section{Effect of the cisplatin applied before and after the surgical procedure on the healing of colonic anastomoses in rats}

\author{
Alvo Orlando Vizzotto Junior ${ }^{1}$ \\ Lucia de Noronha ${ }^{2}$ \\ Darliany Louise H. Scheffe ${ }^{3}$ \\ Antonio Carlos L. Campos
}

\begin{tabular}{|c|c|}
\hline $\mathbf{u}$ & \\
\hline $\begin{array}{l}\text { Cisplatina } \\
\text { Anastomose colônica } \\
\text { Infiltrado neutrofílico } \\
\text { Cicatrização }\end{array}$ & $\begin{array}{l}\text { Introdução: A cisplatina é utilizada como droga de escolha para o tratamento do câncer de } \\
\text { ovário, antes ou depois do procedimento cirúrgico, no qual há freqüentemente a confecção de } \\
\text { uma anastomose intestinal. Objetivo: Avaliação histológica do efeito da cisplatina sobre a } \\
\text { cicatrização das anatomoses colônicas. Material e métodos: Foram utilizados } 80 \text { ratos Wistar } \\
\text { divididos em três grupos: um grupo controle, que foi submetido ao procedimento cirúrgico e } \\
\text { recebeu solução salina nos cinco dias subseqüentes, incluindo o dia da operação; um grupo } \\
\text { que recebeu cisplatina intraperitoneal na dose de } 0,35 \text { mg/kg por cinco dias antes do procedi- } \\
\text { mento cirúrgico; e um grupo que recebeu cisplatina intraperitoneal na mesma dose por cinco } \\
\text { dias depois do procedimento cirúrgico. O procedimento cirúrgico constou de secção da alça } \\
\text { colônica esquerda, } 5 \text { cm acima da reflexão peritoneal, e anastomose em plano único. Metade } \\
\text { dos ratos foi sacrificada no quinto dia de pós-operatório e a outra metade, no oitavo. Foram } \\
\text { avaliados parâmetros histológicos de reação inflamatória com a elaboração de índices e escores } \\
\text { para comparação entre os grupos do estudo. Resultados: A média do índice infiltrado neutrofilico } \\
\text { foi significativamente maior no grupo que recebeu cisplatina depois do procedimento cirúrgico } \\
\text { em relação ao grupo controle e ao grupo que recebeu droga antes do procedimento } \\
\text { cirúrgico. A média do índice intensidade do edema foi significativamente maior nos grupos } \\
\text { que receberam cisplatina em relação ao grupo controle. A análise do escore histológico final } \\
\text { permitiu a classificação do processo inflamatório como subagudo nos grupos que receberam } \\
\text { cisplatina e como crônico no grupo controle. Discussão e conclusão: A cisplatina interfere no } \\
\text { processo de cicatrização das anastomoses colônicas, produzindo uma lentificação do mesmo. }\end{array}$ \\
\hline
\end{tabular}

\section{abstract}

Background: Cisplatin is the first line drug in the treatment of the ovarian cancer. This drug is often applied before or after a surgical procedure to whom a large bowel anastomosis is done. Objective: Histological evaluation of the effect of the cisplatin on the healing of the colonic anastomoses. M ethods: Eighty male Wistar rats were divided in three groups: the control group, the group that received intraperitoneal cisplatin injection for five days before the surgical procedure and the group that received the cisplatin for five days after the surgical procedure. The cisplatin was used in the dose of $0,35 \mathrm{mg} / \mathrm{kg}$. The large bowel was transected $5 \mathrm{~cm}$ above peritoneal reflection and it was anastomosed in one plan suture. Half of the animals were sacrificed on the fifth day and the other half on the eighth day after the surgical procedure. Histological parameters of inflammatory reaction were evaluated with the elaboration of indexes and scores to compare the groups of this study. Results: The average of neutrophilic infiltrating index was significatively bigger in the group that received cisplatin after the surgical procedure when compared with the control group and the group

that received cisplatin before the surgery. The average of intensity of the edema index was significatively bigger in the groups that received cisplatin when compared with the control group. The analysis of the final histological score allowed the classification of the inflammatory process as subacute in the groups that received cisplatin and chronic in the control group. Discussion and conclusion: Cisplatin produces an impairment on the healing of the colonic anastomoses. key words Cisplatin Large bowel anastomosis Neutrophilic infiltrating Healing
1. Mestre em Clínica Cirúrgica. 2. Professora doutora da disciplina de Patologia da Pontifícia Universidade Católica do Paraná (PUC-PR). 3. Acadêmica de iniciação cientifica da PUC-PR.

4. Professor doutor; coordenador do Programa de Pós-Graduação em Clínica Cirúrgica da Universidade Federal do Paraná (UFPR). 


\section{Introdução}

A utilização de cisplatina como terapia adjuvante precoce no carcinoma de ovário é fundamental para se obter maior sobrevida. No entanto esta droga poderia interferir na cicatrização das anastomoses intestinais, freqüentemente necessárias no tratamento cirúrgico do tumor de ovário, como nos casos de oclusão intestinal por implantes. Assim, o conhecimento apurado da interferência da cisplatina na cicatrização das anastomoses intestinais é fundamental para se definir o momento correto para o início do tratamento adjuvante ou os riscos de uma anastomose realizada sob efeito de quimioterapia (10).

Poucos trabalhos encontrados na literatura estudaram experimentalmente a ação da cisplatina na cicatrização das anastomoses intestinais. Roy van Zuidewijn et al. $(7,8)$ investigaram a ação da cisplatina associada à bleomicina e ao 5-fluorouracil na cicatrização de anastomoses colônicas de ratos. No entanto nenhum estudo utilizando apenas cisplatina com administração pré e pósoperatória foi encontrado na revisão da literatura. Desta forma, justifica-se avaliar experimentalmente a interferência deste quimioterápico isolado antes e após o ato cirúrgico sobre a cicatrização das anastomoses colônicas.

A microscopia ótica é um método de avaliação da evolução do processo de cicatrização das anastomoses intestinais utilizado com freqüência nos estudos experimentais. Seu valor é, em geral, subestimado, pois este método permite avaliação de todos os passos do processo cicatricial. O inconveniente consta no fato de que este método é subjetivo. Porém, em mãos experientes que analisem de maneira imparcial, é possível extrair dados passíveis de quantificação, fundamentais para avaliação e comparação do processo de cicatrização (10).

O processo de reparação pode ser avaliado em cortes histológicos corados com hematoxilina-eosina (HE) por diferentes indicadores, tais como: congestão vascular, necrose de mucosa, deposição de fibrina, edema intersticial, exsudato neutrofilico, necrose transmural, atividade regenerativa da mucosa, neoformação vascular, infiltrado monomorfonuclear, proliferação fibroblástica e fibrose intersticial (10).

Sousa (9), Oliveira (6) e Vizzotto (10) utilizaram o parâmetro histológico em seus trabalhos para avaliação do processo de cicatrização de anastomoses colônicas em coelhos e ratos. Ambos estudaram os parâmetros expostos anteriormente, classificando-os e quantificando-os, de acordo com sua presença e intensidade, em ausente, leve, moderado ou intenso.
O objetivo deste estudo é a avaliação histológica do efeito da cisplatina administrada intraperitonealmente no pré e no pós-operatório sobre a cicatrização das anastomoses colônicas em ratos Wistar adultos.

\section{Material e método}

\section{Material}

\section{Animais}

Foram utilizados 80 ratos Wistar (Rattus norvegicus albinus, Roentia Mammalia), do sexo masculino, adultos, com peso corporal variando entre $195 \mathrm{~g}$ e $280 \mathrm{~g}$.

\section{Droga quimioterápica}

A droga utilizada foi a cisplatina [CDDP ou cisdiaminodicloroplatina (Dabur Índia, Badi, Salan, Índia)] na dose de $0,35 \mathrm{mg} / \mathrm{kg} /$ dia em injeção intraperitoneal.

\section{Método}

\section{Grupos}

Os ratos foram divididos em três grupos:

- grupo $\mathrm{C}$ - controle $(n=24)$ - foi submetido ao procedimento cirúrgico e recebeu injeção intraperitoneal de $2 \mathrm{ml}$ de solução salina isotônica a $0,9 \%$ nos cinco dias subseqüentes, incluindo o dia da operação;

- grupo A - antes $(n=24)$ - recebeu injeção intraperitoneal de droga cisplatina por cinco dias e foi operado no dia seguinte ao término do ciclo de quimioterapia;

- grupo $D$ - depois $(n=24)$ - recebeu injeção intraperitoneal de cisplatina por cinco dias, com início no dia do procedimento cirúrgico.

\section{Procedimento cirúrgico}

Pré-operatório: todos os animais foram submetidos a jejum de 12 horas prévias ao procedimento cirúrgico, com o objetivo de diminuir o conteúdo fecal no intestino grosso do rato, local em que foi realizada a anastomose.

Peroperatório: a anestesia foi feita com éter etílico por via inalatória. A seguir procedeu-se à tricotomia abdominal com solução degermante à base de iodo e posterior fixação do animal à mesa cirúrgica com os membros em extensão seguida da anti-sepsia abdominal com polivinilpirrolidona-iodo a $10 \%$. Por meio de uma incisão abdominal mediana de cerca de $4 \mathrm{~cm}$ de extensão obteve- 
se acesso à cavidade abdominal. Procedeu-se à localização do cólon esquerdo e, a cerca de $5 \mathrm{~cm}$ acima da reflexão peritoneal, efetuou-se a secção completa do segmento colônico com dissecção e proteção da arcada vascular. A reconstrução foi feita com anastomose terminoterminal em plano único total por meio de sutura interrompida utilizando-se oito pontos de fio monofilamentar Paralon 6 x 0 (Paramed Suturas, São Paulo, SP, Brasil) em dois planos: musculoaponeurótico e subcuticular, não-ancorados.

\section{Sacrifício}

Metade dos animais de cada grupo foi submetida ao sacrifício no quinto dia de pós-operatório, e a outra metade, no oitavo dia de pós-operatório. O sacrifício foi feito com dose letal de éter etílico inalatório. Após isto realizou-se laparotomia mediana com acesso à cavidade abdominal. Na seqüência, após inspeção da cavidade abdominal, procedeu-se à retirada de um segmento de $4 \mathrm{~cm}$ de comprimento de intestino grosso contendo a anastomose na porção média.

Os ratos do grupo controle que foram submetidos ao sacrifício no quinto dia de pós-operatório foram denominados C5, e os do oitavo dia de pós-operatório foram denominados C8. Da mesma maneira, os animais que receberam cisplatina antes do procedimento cirúrgico foram denominados $A 5$ e A8, e os animais que receberam cisplatina depois do ato cirúrgico foram denominados D5 e D8.

Houve cinco óbitos no pós-operatório: dois do grupo controle, dois do grupo que recebeu droga depois do procedimento cirúrgico e um do grupo que recebeu droga antes do ato operatório. Além disso, foram excluídos quatro animais devido a problemas técnicos que impossibilitaram a análise histológica. No final, os grupos foram compostos da seguinte maneira: C5 com 11 animais, C8 com dez animais, A5 com 11 animais, A8 com 11 animais, D5 com dez animais e D8 com dez animais.

\section{Avaliação histológica}

O material foi fixado em formalina a $10 \%$ e processado rotineiramente para avaliação histológica. Os cortes histológicos foram feitos com espessura de 4 micrômetros e corados com HE (3). Avaliaram-se tipo e quantidade das células predominantes na reação inflamatória (infiltrados poli e monomorfonuclear), presença de edema intersticial e congestão vascular e o grau de formação de tecido de granulação e de fibrose (1).

Os dados foram classificados em acentuado, moderado, discreto e ausente, de acordo com a intensidade em que foram encontrados, e transformados em variáveis quantitativas através da atribuição de índice aos achados histológicos. O edema, a congestão e o exsudato neutrofílico foram indicativos de processo inflamatório agudo, e o tecido de granulação, a fibrose e o exsudato monomorfonuclear foram achados indicativos de processo inflamatório crônico. Aos índices do processo inflamatório agudo foi atribuído sinal negativo, e aos índices do processo inflamatório crônico foi atribuído sinal positivo. A atribuição dos índices ocorreu como mostra a Tabela 1.

Após a atribuição dos índices, procedeu-se à somatória destes, de maneira que cada grupo de animais teve um escore final, permitindo assim a classificação dos grupos em três fases do processo inflamatório, conforme a Tabela 2.

Os animais de cada grupo receberam os índices de valores dos achados histológicos, tendo sido calculados a média e o desvio padrão de cada um destes achados, conforme apresentado nas Tabelas 3 e 4.

A avaliação histológica foi feita sem o conhecimento do grupo a que pertenciam os animais. Desta forma, nem o cirurgião nem o patologista tinham conhecimento de

Classificação e atribuição de índices aos achados histológicos de

Tabela 1 hematoxilina-eosina (HE)

\begin{tabular}{lcccc}
$\begin{array}{l}\text { Parâmetros } \\
\text { inflamatórios }\end{array}$ & Acentuada & Moderada & Discreta Ausente \\
& -3 & -2 & -1 & 0 \\
Neutrófilos & -3 & -2 & -1 & 0 \\
Edema & -3 & -2 & -1 & 0 \\
Congestão & 3 & 2 & 1 & 0 \\
Monomorfonucleares & 3 & 2 & 1 & 0 \\
Tecido de granulação & 3 & 2 & 1 & 0 \\
Fibrose & 3 & & & \\
\hline
\end{tabular}

Caracterização da fase do processo inflamatório de acordo com o escore Tabela 2 final de cada grupo

$\begin{array}{lc}\begin{array}{l}\text { Fase do processo } \\ \text { inflamatório }\end{array} & \begin{array}{c}\text { Escore final de } \\ \text { classificação }\end{array} \\ \text { Agudo } & -9 \text { a - } 3 \\ \text { Subagudo } & -3,1 \text { a } 3 \\ \text { Crônico } & 3,1 \text { a } 9\end{array}$


qual era o animal a ser avaliado, evitando-se assim a indução de resultados.

\section{Análise estatística}

Os dados da avaliação histológica foram submetidos a análise de variância de médias (Anova), utilizando-se as metodologias de análise paramétrica e análise nãoparamétrica para a comparação dos dados.

\section{Resultados}

Os resultados dos achados histológicos estão apresentados de acordo com a divisão em três e seis subgrupos. A primeira análise levou em consideração a divisão em seis subgrupos, de acordo com o momento do recebimento da cisplatina ou da solução salina e de acordo com o dia do sacrifício (C5, C8, A5, A8, D5 e D8), como apresentado na Tabela 3.

No grupo que recebeu cisplatina antes do ato cirúrgico (A) houve redução significativa do exsudato neutrofílico do quinto para o oitavo dia $(p<0,01)$. Entre os grupos controles C5 e C8, e entre os grupos que receberam cisplatina depois do procedimento cirúrgico, D5 e D8, não houve diferença significativa com relação ao infiltrado neutrofílico $(p>0,05)$.

$\mathrm{O}$ índice de edema foi de 0,63 no grupo controle C5 e de 0,9 no grupo controle $C 8$, o que sugere um aumento do edema do quinto para o oitavo dia de sacrifício, embora não tenha sido estatisticamente significativo. Com relação aos grupos que receberam cisplatina antes (A5 e A8) e depois (D5 e D8) do ato cirúrgico, não foram observadas diferenças na avaliação da presença de edema.
A média do infiltrado mononuclear só teve diferença significativa entre os grupos que receberam a droga depois do procedimento cirúrgico, sendo que em D5 foi de 2,4 e em D8 foi de $2(p<0,05)$.

O tecido de granulação apresentou índice médio de 1,81 e 2,5 nos grupos controles ( $C 5$ e C8) $(p=0,126)$, de 1,9 e 2,9 nos grupos que receberam cisplatina antes do ato cirúrgico (A5 e A8) $(p<0,0001)$ e de 1,8 e 2,7 nos grupos que receberam cisplatina depois do procedimento cirúrgico (D5 e D8) $(p=0,0061)$, respectivamente.

A intensidade de fibrose obteve índice médio de 1,81 e 2,5 nos grupos controles (C5 e C8) $(p=0,0822)$, de 1,54 e 2,36 nos grupos que receberam cisplatina antes do ato cirúrgico (A5 e A8) $(p=0,0043)$ e de 1,6 e 2,5 nos grupos que receberam cisplatina depois do procedimento cirúrgico (D5 e D8) $(p=0,001)$, respectivamente.

Os resultados de histologia, na segunda análise, foram divididos em três subgrupos [grupo controle $(C)$, grupo que recebeu cisplatina antes do ato cirúrgico $(A)$ e grupo que recebeu cisplatina depois do ato cirúrgico (D)], como mostra a Tabela 4

O infiltrado neutrofílico foi significativamente mais intenso no grupo que recebeu droga depois do procedimento cirúrgico (D) em relação ao grupo que recebeu droga antes do procedimento cirúrgico $(A)$ e ao grupo controle $(C)(p=0,0005)$.

A intensidade do edema foi significativamente maior nos grupos que receberam cisplatina ( $A$ e $D)$ em relação ao grupo controle (C) $(p=0,004)$.

A média dos índices do infiltrado monomorfonuclear foi de 2 no grupo que recebeu cisplatina antes do procedimento cirúrgico (A), de 2,04 no grupo controle (C) e de

Média e desvio padrão dos índices histológicos nos grupos controles (C5 e C8), nos grupos que receberam cisplatina antes do ato cirúrgico (A5 e A8) e nos grupos que receberam Tabela 3 cisplatina depois do procedimento cirúrgico (D5 e D8)

\begin{tabular}{ccccccc}
\hline $\begin{array}{c}\text { Grupo } \\
\text { (n) }\end{array}$ & $\begin{array}{c}\text { Neutro } \\
(\mu \pm \mathrm{DP})\end{array}$ & $\begin{array}{c}\text { Edema } \\
(\mu \pm \mathrm{DP})\end{array}$ & $\begin{array}{c}\text { Cong, } \\
(\mu \pm \mathrm{DP})\end{array}$ & $\begin{array}{c}\text { Mono } \\
(\mu \pm \mathrm{DP})\end{array}$ & $\begin{array}{c}\text { Tec gran, } \\
(\mu \pm \mathrm{DP})\end{array}$ & $\begin{array}{c}\text { Fibrose } \\
(\mu \pm \mathrm{DP})\end{array}$ \\
C5 (11) & $-1,27 \pm 0,46$ & $-0,63 \pm 0,5$ & $-1 \pm 0$ & $2 \pm 0$ & $1,81 \pm 0,98$ & $1,81 \pm 0,87$ \\
C8 (10) & $-1,3 \pm 0,48$ & $-0,9 \pm 0,56$ & $-1 \pm 0,47$ & $2,1 \pm 0,56$ & $2,5 \pm 0,85$ & $2,5 \pm 0,85$ \\
A5 (11) & $-1,72 \pm 0,74$ & $-1 \pm 0$ & $-1 \pm 0$ & $2 \pm 0$ & $1,9 \pm 0,53$ & $1,54 \pm 0,68$ \\
A8 (11) & $-1 \pm 0$ & $-1 \pm 0$ & $-1 \pm 0$ & $2 \pm 0$ & $2,9 \pm 0,3$ & $2,36 \pm 0,5$ \\
D5 (10) & $-1,8 \pm 0,42$ & $-1 \pm 0$ & $-1 \pm 0$ & $2,4 \pm 0,51$ & $1,8 \pm 0,78$ & $1,6 \pm 0,51$ \\
D8 (10) & $-1,7 \pm 0,48$ & $-1 \pm 0$ & $-1 \pm 0$ & $2 \pm 0$ & $2,7 \pm 0,48$ & $2,5 \pm 0,52$ \\
\hline
\end{tabular}

Neutro = infiltrado neutrofilico; cong. = congestão; mono = infiltrado monomorfonuclear; tec. gran. = tecido de granulação; $n=n$ o de animais; $\mu=$ média; DP = desvio padrão. 


\section{Média e desvio padrão dos índices histológicos no grupo controle $(C)$, no grupo que} recebeu cisplatina antes do ato cirúrgico (A) e no grupo que recebeu cisplatina depois

\section{Tabela 4}

\begin{tabular}{ccccccc}
\hline $\begin{array}{c}\text { Grupo } \\
\text { (n) }\end{array}$ & $\begin{array}{c}\text { Neutro } \\
(\mu \pm \mathrm{DP})\end{array}$ & $\begin{array}{c}\text { Edema } \\
(\mu \pm \mathrm{DP})\end{array}$ & $\begin{array}{c}\text { Cong. } \\
(\mu \pm \mathrm{DP})\end{array}$ & $\begin{array}{c}\text { Mono } \\
(\mu \pm \mathrm{DP})\end{array}$ & $\begin{array}{c}\text { Tec gran. } \\
(\mu \pm \mathrm{DP})\end{array}$ & $\begin{array}{c}\text { Fibrose } \\
(\mu \pm \mathrm{DP})\end{array}$ \\
$\mathrm{C}(21)$ & $-1,28 \pm 0,46$ & $-0,76 \pm 0,53$ & $-1 \pm 0,31$ & $2,04 \pm 0,38$ & $2,14 \pm 0,96$ & $2,14 \pm 0,91$ \\
A (22) & $-1,36 \pm 0,58$ & $-1 \pm 0$ & $-1 \pm 0$ & $2 \pm 0$ & $2,4 \pm 0,66$ & $1,95 \pm 0,72$ \\
D(22) & $-1,75 \pm 0,44$ & $-1 \pm 0$ & $-1 \pm 0$ & $2,2 \pm 0,41$ & $2,25 \pm 0,78$ & $2,05 \pm 0,68$ \\
\hline
\end{tabular}

Neutro = infiltrado neutrofilico; cong. = congestão; mono = infiltrado monomorfonuclear; tec. gran. = tecido de granulação; $n=n=$ de animais; $\mu=$ média; DP = desvio padrão.

2,2 no grupo que recebeu cisplatina depois do procedimento cirúrgico (D). O tecido de granulação apresentou índices médios de 2,14; 2,25 e 2,4 nos grupos C, D e A, respectivamente. $A$ intensidade da fibrose apresentou índices médios de 1,95; 2,05 e 2,14 para os grupos A, D e $C$, respectivamente. Não houve diferença significativa entre os grupos que receberam cisplatina e o grupo controle para estes três últimos achados histológicos.

$\mathrm{Na}$ análise do escore final podemos observar que os grupos C5, A5 e D5 apresentaram processo inflamatório subagudo, pois suas médias finais foram 2,72; 1,72 e 2, respectivamente. Já os grupos C8, A8 e D8 foram classificados como portadores de processo inflamatório crônico, pois suas médias finais foram de 3,9; 4,27 e 3,5, respectivamente (Tabela 5).

Ao se analisar os achados histológicos de acordo com a divisão em três subgrupos, observou-se que os grupos que receberam cisplatina antes do ato cirúrgico $(A)$ e depois do ato cirúrgico (D) apresentaram médias finais de 3 e $2,75(p=0,5958)$, respectivamente, tendo sido classificados como subagudos. O grupo controle (C) apresentou média de 3,28 e foi classificado como crônico.

\section{Discussão}

O método histológico é utilizado com grande freqüência como meio de avaliação da evolução da cicatrização das anastomoses. Embora tenha seu valor questionável pela subjetividade na sua obtenção, em mãos experientes e sendo submetido à padronização dos achados em índices de intensidade, o método histológico pode ser de grande valor na avaliação do processo cicatricial (10).

Da mesma forma como proposto por Sousa (9) e Oliveira (6), neste estudo foi utilizado o parâmetro histológico com atribuição de índices de intensidade de acordo com os achados histológicos.

O infiltrado neutrofílico é o principal achado histológico na fase aguda do processo cicatricial e é o que o caracteriza como agudo. A quantidade de neutrófilos no leito da ferida tem íntima relação com o processo de proliferação, ativação e movimentação celular, bem como com a produção de citocinas, que são as proteínas mediadoras do processo inflamatório. Os outros achados do processo inflamatório agudo, como edema intersticial e congestão vascular, têm ligação menos estreita com o processo de

Classificação do processo inflamatório de acordo com 0 escore histológico final [grupos controles (C5 e C8), grupos que receberam cisplatina antes do ato cirúrgico (A5 e A8) e Tabela 5 grupos que receberam cisplatina depois do procedimento cirúrgico (D5 e D8)]

\begin{tabular}{ccc}
\hline Grupo & Fase do processo inflamatório & Média dos escores finais do PI $(\mu \pm$ DP) \\
C5 & Subagudo & $2,72 \pm 1,61$ \\
A5 & Subagudo & $1,72 \pm 1,42$ \\
D5 & Subagudo & $2 \pm 0,81$ \\
C8 & Crônico & $3,9 \pm 1,52$ \\
A8 & Crônico & $4,27 \pm 0,64$ \\
D8 & Crônico & $3,5 \pm 0,26$ \\
\hline
\end{tabular}

$\mathrm{PI}=$ processo inflamatório; $\mathrm{DP}=$ desvio padrão. 
proliferação celular. Já o processo inflamatório crônico está associado histologicamente à presença de infiltrado monomorfonuclear, tecido de granulação e fibrose (1).

Ikeuchi (4) propôs que a fase inicial do processo cicatricial terminaria por volta do quarto dia de pós-operatório e, desta forma, a partir do quinto dia de pós-operatório, seria esperado que o infiltrado polimorfonuclear (neutrofílico) fosse menos intenso, dando lugar ao infiltrado monomorfonuclear e aos fibroblastos.

Fumagalli (2) e Kanellos (5), avaliando mitomicina no primeiro e 5-fluorouracil no segundo, encontraram persistência dos achados histológicos de processo inflamatório agudo nos animais que receberam a droga quimioterápica. Propuseram que haveria retardo no processo de reparação das anastomoses colônicas com persistência da fase proliferativa.

Roy van Zuidewijn et al. $(7,8)$ avaliaram a interferência da cisplatina associada à bleomicina e ao 5 -fluorouracil na cicatrização de anastomoses colônicas de ratos. Estes autores observaram redução significativa da quantidade de hidroxiprolina, que reflete a produção de colágeno na anastomose, nos grupos que receberam quimioterapia em relação ao grupo controle, e ainda, que esta diminuição foi dose-dependente, isto é, quanto maior a dose do quimioterápico, maior a diminuição (Quadro). A interferência das drogas quimioterápicas sobre a cicatrização destas anastomoses é congruente com os achados do nosso estudo.
Observa-se, no presente estudo, que as diferenças existentes entre os grupos avaliados com relação aos dias de sacrifício 5 e 8 refletem o desenvolvimento do processo inflamatório normal, sendo que o dia 5 representa a fase subaguda do mesmo, e o dia 8, a fase crônica, pois sabese que durante a fase subaguda ocorre redução do infiltrado neutrofílico e a partir do final da mesma inicia-se o processo de reparo, caracterizando o início da fase crônica na qual ocorre aumento do tecido de granulação e fibrose. Assim demonstra-se que não houve influência da cisplatina na evolução do processo inflamatório do dia 5 para o 8, sugerindo que a influência da cisplatina possa ter ocorrido na fase aguda do processo inflamatório, apenas lentificando-o.

Por isso, numa segunda análise, os grupos que primeiramente foram divididos entre os dias de sacrifício 5 e 8 foram reunidos e avaliados em conjunto (C, A e D).

O grupo que recebeu cisplatina depois do procedimento cirúrgico (D) apresentou índice de infiltrado neutrofílico significativamente maior $(p=0,0005)$ que $o$ grupo que recebeu esta droga antes do ato operatório (A) e o grupo controle (C). Além disso, o edema intersticial foi mais intenso nos grupos que receberam cisplatina antes (A) e depois (D) do procedimento cirúrgico em relação ao grupo controle (C). Como o edema intersticial e o infiltrado neutrofílico são achados histológicos característicos da fase aguda da inflamação, este dado sus-

\section{Quadro Resultados obtidos nos estudos de Roy van Zuidewijn}

\begin{tabular}{|c|c|c|c|}
\hline & $\begin{array}{l}\text { Drogas, doses ( } \mathrm{mg} / \mathrm{kg} \text { ) } \\
\text { e via de administração }\end{array}$ & Variáveis & Resultados \\
\hline Roy van Zuidewijn et al. (1986) & $\begin{array}{l}\text { Bleomicina } 2 \mathrm{EV} \\
\text { Cisplatina 0,35 EV } \\
\text { 5-fluorouracil } 10 \mathrm{EV}\end{array}$ & $\begin{array}{l}\text { Quantidade de } \\
\text { hidroxiprolina } \\
\text { na anastomose }\end{array}$ & $\begin{array}{l}\text { Redução significativa da } \\
\text { dosagem de hidroxiprolina } \\
\text { nos grupos submetidos a } \\
\text { quimioterapia em relação ao } \\
\text { grupo controle }\end{array}$ \\
\hline Roy van Zuidewijn et al. (1991) & $\begin{array}{l}\text { 10 Grupo: } \\
\text { Bleomicina } 2 \text { IP } \\
\text { Cisplatina 0,35 IP } \\
\text { 5-fluorouracil } 10 \text { IP } \\
\text { 20 Grupo: } \\
\text { Bleomicina } 4 \text { IP } \\
\text { Cisplatina 0,70 IP } \\
\text { 5-fluorouracil } 20 \text { IP }\end{array}$ & $\begin{array}{l}\text { Quantidade de } \\
\text { hidroxiprolina na } \\
\text { anastomose }\end{array}$ & $\begin{array}{l}\text { Diminuição significativa } \\
\text { e dose-dependente da } \\
\text { quantidade de hidroxiprolina } \\
\text { nas anastomoses nos } \\
\text { grupos que receberam } \\
\text { quimioterapia }\end{array}$ \\
\hline
\end{tabular}

$\mathrm{EV}=$ endovenosa; $\mathrm{IP}$ = intraperitoneal. 
cita a idéia de que a cisplatina interfira no processo inflamatório agudo produzindo uma lentificação do mesmo, o que é congruente com os relatos de Fumagalli (2) e Kanellos (5). Outro fator que corrobora esta idéia é que o grupo controle se apresentou na fase crônica da reação inflamatória, enquanto que os grupos que receberam cisplatina ( $A$ e $D$ ) foram classificados como estando na fase subaguda deste processo. Tal fato também é reforçado pelos resultados obtidos com a intensidade do infiltrado monomorfonuclear, da fibrose e do tecido de granulação, achados típicos do processo inflamatório crônico, os quais não apresentaram diferenças entre 0 grupo controle $\mathrm{C}$ e o experimento.

\section{Conclusão}

Com relação à utilização da cisplatina administrada intraperitonealmente no pré e no pós-operatório sobre a cicatrização das anastomoses colônicas em ratos Wistar adultos, podemos concluir que, do ponto de vista histológico, a cisplatina produz índice significativamente maior de infiltrado neutrofílico e edema intersticial no leito da anastomose em relação ao grupo controle. Tal fato, reforçado pelos índices e escores obtidos, sugere que a cisplatina possa interferir na fase aguda da reação inflamatória, produzindo uma lentificação no processo cicatricial, principalmente quando aplicada depois do ato cirúrgico.

\section{Referências}

1.Cotran, R.S.; Kumar,V.\& Collins,T.A cute and chronic inflammation. In:C otran, R.S.; Kumar,V. \& C ollins,T.6. ed. Robbins pathologic basis of disease. Philadelphia:W . B. Saunders, 1999. p. 5088.

2. Fumagalli, U. et al. Effect of intraperitoneal chemotherapy on anastomostic healing in the rat.J. Surg. Res., 50:82-7, 1991.

3. Gordon, K.C . \& Bradbury, P. M icrotomy and paraffin sections. In: Bancroft,J.D .\& Stevens,A .Theory and practice of histological techniques. London: Churchill Livinsgstone, 1982, p. 61-81.

4. Ikeuchi, D. et al. Correlation of tensile strength with bursting pressure in the evaluation of intestinal anastomosis. Dig. Surg., 16: 478-85, 1999.

5. Kanellos, I. et al. Influence of intraperitoneal 5-fluorouracil plus folinic acid on the healing of colonic anastomosis in rats. Eur. Sur. Res., 28: 374-9, 1996.

6. 0 liveira, P. G . Efeito da peritonite por C andida albicans na cicatrização de anastomoses colônicas: estudo experimental em ratos. Ribeirão Preto, 1995.Tese (doutoramento), Faculdade Médica de Ribeirão Preto, Universidade de São Paulo.

7. Roy van Zuidewijn, D.B.W . de et al.The effect of antineo plastic agents on the healing of small intestinal anastomosis in the rat. Cancer, Philadelphia, 58:62-6, 1986.

8. Roy van Zuidewijn, D. B.W . de et al. Intraperitoneal cytostatics impair healing of experimental intestinal anastomosis. Br.J. Cancer, 63: 937-41, 1991.

9. Sousa, J. B. Evolução da cicatrização de anastomoses colônicas sob a ação do diclofenaco sódico administrado no período perioperatório: estudo experimental em coelhos. Ribeirão Preto, 1994. Tese (doutoramento), Faculdade Médica de Ribeirão Preto, Universidade de São Paulo.

10. Vizzotto, 0 . Influência da cisplatina administrada no pré e no pós-operatório sobre a cicatrização de anastomoses colônicas em ratos testada pela força de resistência à tração. C uritiba, 2001. Tese (mestrado), U niversidade Federal do Paraná.
Endereco para correspondência

Lúcia de Noronha

Laboratório de Patologia Experimental

Centro de Ciências Biológicas e da Saúde - Campus 1

Pontifícia Universidade Católica do Paraná

Rua Imaculada Conceição 1155 - Prado Velho

CEP 80215-901 - Curitiba-PR

Tel.: (41) 330-1515, ramal 2264

Fax: (41) 330-1624 